\title{
Methotrexate and Sulfasalazine Promote Adenosine Release by a Mechanism that Requires Ecto-5'-nucleotidase-mediated Conversion of Adenine Nucleotides
}

\author{
Lance Morabito, ${ }^{\star}$ M. Carmen Montesinos, ${ }^{*}$ David M. Schreibman, ${ }^{\star}$ Laurence Balter, ${ }^{\star}$ Linda F. Thompson, ${ }^{\ddagger}$ Regina Resta, ${ }^{\ddagger}$ \\ Gunnar Carlin, ${ }^{\S}$ Mary Ann Huie, ${ }^{*}$ and Bruce N. Cronstein* \\ *Department of Medicine, New York University Medical Center, 550 First Avenue, New York, New York 10016; ${ }^{\ddagger}$ Immunobiology and \\ Cancer Program, Oklahoma Medical Research Foundation, Oklahoma City, Oklahoma 73104; and ${ }^{\S}$ Pharmacia-Upjohn, Inc., \\ Pharmaceuticals Uppsala, Department of Pharmacology, S-751-82 Uppsala, Sweden
}

\begin{abstract}
We and others have shown that an increased extracellular concentration of adenosine mediates the antiinflammatory effects of methotrexate and sulfasalazine both in vitro and in vivo, but the mechanism by which these drugs increase extracellular adenosine remains unclear. The results of the experiments reported here provide three distinct lines of evidence that adenosine results from the ecto-5' -nucleotidasemediated conversion of adenine nucleotides to adenosine. First, pretreatment of a human microvascular endothelial cell line (HMEC-1) with methotrexate increases extracellular adenosine after exposure of the pretreated cells to activated neutrophils; the ecto-5' -nucleotidase inhibitor $\alpha, \beta$-methylene adenosine-5' -diphosphate (APCP) abrogates completely the increase in extracellular adenosine. Second, there is no methotrexate-mediated increase in extracellular adenosine concentration in the supernate of cells deficient in ecto-5' -nucleotidase, but there is a marked increase in extracellular adenosine concentration in the supernates of these cells after transfection and surface expression of the enzyme. Finally, as we have shown previously, adenosine mediates the antiinflammatory effects of methotrexate and sulfasalazine in the murine air pouch model of inflammation, and injection of APCP, the ecto-5'-nucleotidase inhibitor, abrogates completely the increase in adenosine and the decrement in inflammation in this in vivo model. These results not only show that ecto-5'-nucleotidase activity is a critical mediator of methotrexate- and sulfasalazine-induced antiinflammatory activity in vitro and in vivo but also indicate that adenine nucleotides, released from cells, are the source of extracellular adenosine. (J. Clin. Invest. 1998. 101:295-300.) Key words: adenosine - ecto-5'nucleotidase $\bullet$ methotrexate $\bullet$ sulfasalazine $\bullet$ inflammation
\end{abstract}

\section{Introduction}

We have demonstrated previously in both in vitro and in vivo studies that the antiinflammatory properties of low-dose methotrexate and sulfasalazine are mediated by adenosine, a potent

Address correspondence to Dr. Bruce N. Cronstein, Department of Medicine, New York University Medical Center, 550 First Avenue, New York, NY 10016. Phone: 212-263-6404; FAX: 212-263-8804; E-mail: cronsb01@mcrcr6.med.nyu.edu

Received for publication 20 August 1997 and accepted in revised form 17 November 1997.

J. Clin. Invest.

(C) The American Society for Clinical Investigation, Inc. 0021-9738/98/01/0295/06 \$2.00

Volume 101, Number 2, January 1998, 295-300

http://www.jci.org antiinflammatory autocoid (1-3). Methotrexate and sulfasalazine promote adenosine release from a variety of different cell types and tissues, particularly when the cells or tissues are undergoing a physiologic stress $(1,3)$. The adenosine released at inflamed sites interacts with specific receptors on inflammatory cells to diminish inflammation and tissue injury (for a review see reference 4).

The mechanism by which methotrexate and sulfasalazine promote adenosine release is not well understood. Both methotrexate and sulfasalazine are taken up by cells where they or their metabolites inhibit 5-aminoimidazole-4-carboxamide ribonucleotide (AICAR) ${ }^{1}$ transformylase (5-7). The two- to threefold increase in intracellular (splenocyte) AICAR concentration in animals treated with pharmacologically relevant doses of methotrexate or sulfasalazine is consistent with the hypothesis that low-dose methotrexate treatment leads to inhibition of AICAR transformylase in vivo $(2,3)$. Intracellular AICAR accumulation has been associated with adenosine release (8-10), although how and whether intracellular AICAR accumulation promotes adenosine release have not been established.

One potential explanation for the effect of AICAR accumulation on adenosine release is that AICAR inhibits AMP deaminase, thereby increasing intracellular AMP, which may be dephosphorylated either intracellularly or extracellularly to adenosine. Alternatively, accumulated AICAR may be dephosphorylated to its ribonucleoside, an inhibitor of adenosine deaminase, an enzyme that irreversibly deaminates adenosine and deoxyadenosine to inosine and deoxyinosine.

To begin to understand the molecular mechanism by which methotrexate treatment leads to adenosine release, we tested the hypothesis that the adenosine released from stressed cells and tissues after treatment with methotrexate and sulfasalazine is derived from the extracellular dephosphorylation of adenine nucleotides rather than the direct release of adenosine. We report here evidence from both in vitro and in vivo experiments that the methotrexate- and sulfasalazine-mediated increase in extracellular adenosine is accounted for completely by the extracellular generation of adenosine from adenine nucleotides via the ecto-5' -nucleotidase-catalyzed dephosphorylation of AMP.

\section{Methods}

Materials. $\alpha, \beta-$ Methylene adenosine-5'-diphosphate (APCP) and carrageenan were obtained from Sigma Chemical Co. (St. Louis,

1. Abbreviations used in this paper: AICAR, 5-aminoimidazole-4-carboxamide ribonucleotide; APCP, $\alpha, \beta$-methylene adenosine- 5 '-diphosphate; HBS, Hepes-buffered saline; HMEC-1, human microvascular endothelial cell line 1; LDH, lactate dehydrogenase. 
MO). All tissue culture media and reagents were purchased from GIBCO BRL (Gaithersburg, MD). Methotrexate was obtained from Immunex Corp. (San Juan, Puerto Rico). All other reagents were of the highest quality obtainable.

Isolation of leukocytes. Human neutrophils were isolated from whole blood after centrifugation through Hypaque-Ficoll gradients, sedimentation through dextran $(6 \% \mathrm{wt} / \mathrm{vol})$, and hypotonic lysis of red blood cells. Neutrophils were suspended in Hepes-buffered saline (HBS) supplemented with $\mathrm{Mg}^{2+}$ and $\mathrm{Ca}^{2+}$ and counted before addition to confluent human microvascular endothelial cell line 1 (HMEC-1) monolayers (11).

Cell culture. HMEC-1 (obtained from the Centers for Disease Control and Prevention) was cultured in 96-well plates in $200 \mu \mathrm{l}$ of MCDB 131 supplemented with 10\% FBS, 3\% 200 mM L-glutamine, $1 \%$ penicillin/streptomycin in a $5 \% \mathrm{CO}_{2}$ atmosphere at $37^{\circ} \mathrm{C}$. Subconfluent (60-70\% confluent) monolayers were then washed twice with fresh medium and incubated for $48 \mathrm{~h}$ at $37^{\circ} \mathrm{C}$ and $5 \% \mathrm{CO}_{2}$ atmosphere in fresh medium alone or medium containing methotrexate $(100 \mathrm{nM})$. Both methotrexate-treated and control HMEC-1 cells reached confluence under these conditions by the time experiments with neutrophils were performed.

293T cells, a human renal carcinoma cell line transfected with large $\mathrm{T}$ antigen, deficient for ecto-5'-nucleotidase (CD73 [12]) were grown to $60-70 \%$ confluence (before transfection and treatment with methotrexate) on 6-well plates in $4 \mathrm{ml}$ DME supplemented with $10 \%$ FCS, 3\% $200 \mathrm{mM}$ L-glutamine, and $1 \%$ penicillin/streptomycin at $37^{\circ} \mathrm{C}$ in a humidified atmosphere of $5 \% \mathrm{CO}_{2}$ in air. Monolayers were then washed, and medium was replaced with either fresh medium alone or medium containing methotrexate $(100 \mathrm{nM})$. Cells were then grown to confluence over $48 \mathrm{~h}$. Under these conditions, methotrexate did not diminish cell proliferation, and the 293T cells grew to confluence in medium with or without methotrexate.

Stimulation of confluent HMEC-1 with PMNs in the presence of $A P C P$. Monolayers of HMEC-1 cells grown to confluence in 96-well plates were washed twice with medium. To each well were then added, sequentially, $50 \mu \mathrm{l}$ of HBS containing APCP $(50 \mu \mathrm{M})$ or HBS alone, $100 \mu \mathrm{l}$ of HBS containing $1.5 \times 10^{5}$ PMNs, followed by $50 \mu \mathrm{l}$ of either HBS alone or HBS containing the chemotactic peptide $\mathrm{N}$-formyl-methionyl-leucyl-phenylalanine (FMLP, $100 \mathrm{nM}$ ). Plates were then incubated for $2 \mathrm{~h}\left(37^{\circ} \mathrm{C}, 5 \% \mathrm{CO}_{2}\right)$.

Collection of supernatants and pouch exudates for quantitation of adenosine by HPLC. Aliquots of pouch exudates or cell culture supernates were added to a similar volume of TCA $(10 \% \mathrm{vol} / \mathrm{vol})$ followed by extraction of the organic acid with freon/trioctylamine $(77.5 / 22.5 \%, \mathrm{vol} / \mathrm{vol})$. The adenosine concentration of the supernatants was determined by reverse-phase HPLC, as we have described previously (13). Briefly, samples were applied to a $\mu$ BondapackC18 column (Waters Corp., Milford, MA) and eluted with a $0-40 \%$ linear gradient (formed over $60 \mathrm{~min}$ ) of $0.01 \mathrm{M}$ ammonium phosphate $(\mathrm{pH}$ 5.5 ) and methanol, with a $1.5 \mathrm{ml} / \mathrm{min}$ flow rate. Adenosine was identified by retention time and the characteristic ultraviolet ratio of absorbance at 250/260, and the concentration was calculated by comparison to standards. In some experiments, the adenosine peak was digested by treatment with adenosine deaminase $(0.15 \mathrm{IU} / \mathrm{ml}, 30 \mathrm{~min}$ at $37^{\circ} \mathrm{C}$ ) to confirm that the peak so identified contains only adenosine (14). Preliminary studies demonstrated that $90 \%$ of added adenosine is recovered using this technique.

Expression vectors and transfection of CD73 into 2937 cells. The $\mathrm{p} \beta^{\mathrm{NT}}$ expression vector was constructed as described and contains a cDNA for $\operatorname{CD} 73(15,16)$. The empty expression vector, $\mathrm{p} \beta^{\text {neo }}$, which lacks the CD73 cDNA insert, was used as a negative control (sham transfection). The expression vectors for $\mathrm{p} \beta^{\mathrm{NT}}$ and $\mathrm{p} \beta^{\text {neo }}$ were transformed into DaH5 Escherichia coli, and positive clones were selected by ampicillin resistance and restriction enzyme digest. Large scale plasmid preparations were grown and purified following the instructions provided in a commercial kit (QIAGEN Inc., Chatsworth, CA). Medium was removed from subconfluent 293T fibroblasts on 6-well plates and replaced with $3.7 \mathrm{ml}$ DME containing either methotrexate
$(100 \mathrm{nM})$ or medium alone, supplemented with $10 \% \mathrm{FCS}, 3 \% 200 \mathrm{mM}$ L-glutamine, and $1 \%$ penicillin/streptomycin. After a 4-h incubation at $37^{\circ} \mathrm{C}$ in a humidified atmosphere of $5 \% \mathrm{CO}_{2}$, cells were transfected with $300 \mu l$ of either a $\mathrm{p} \beta^{\mathrm{NT}}$ or $\mathrm{p} \beta^{\text {neo }}$ plasmid DNA calcium phosphate solution ( $5 \mu \mathrm{g}$ of plasmid per $4 \mathrm{ml}$ of medium) via the calcium phosphate precipitation method (17). This medium was removed after a 12-16-h incubation period and replaced with fresh medium or medium in the presence of methotrexate. Cells were incubated for an additional $24 \mathrm{~h}$, for a total of 44-48 h of methotrexate treatment (15).

Stimulation of 2937 fibroblasts with $100 \mu M$ hydrogen peroxide, and collection of supernates for adenosine determination. Transfected cells were washed twice with HBS and were then treated with $100 \mu \mathrm{M}$ $\mathrm{H}_{2} \mathrm{O}_{2}$ in Hepes or Hepes alone for $1 \mathrm{~h}$ at $37^{\circ} \mathrm{C}$ and $5 \% \mathrm{CO}_{2}$. Supernatants were collected for HPLC analysis as described above. Neither transfection, methotrexate treatment, $\mathrm{H}_{2} \mathrm{O}_{2}$, nor their combination altered cell viability, as determined by release of lactate dehydrogenase (LDH), as we have described previously (13).

Immunofluorescence. Surface expression of ecto-5'-nucleotidase (CD73) was determined after immunofluorescent labeling by flow cytometry (FACScan ${ }^{\circledR}$; Becton Dickinson, Mountain View, CA) using techniques we have described previously (18). Briefly, cells were stripped from their substrate after incubation in EDTA $(0.01 \% \mathrm{wt} /$ vol) in PBS followed by scraping with a rubber policeman. Cells were washed and then resuspended $\left(2-4 \times 10^{6}\right.$ in a final volume of $\left.50 \mu \mathrm{l}\right)$ in PBS or PBS containing $25 \mu \mathrm{g} / \mathrm{ml}$ anti-CD73 (1E9 [15]) or FLOPC-21 (a murine $\mathrm{mAb}$ directed against an irrelevant antigen). Labeled cells were then resuspended in $1 \mathrm{ml}$ of PBS/1\% sodium azide, washed, and then labeled by incubation in the presence of purified goat antimouse $\mathrm{IgG}_{3}$ labeled with phycoerythrin (Southern Biotechnology Associates, Inc., Birmingham, AL). Labeling was quantitated by flow cytometry $\left(\right.$ FACScan $\left.{ }^{\circledR}\right)$.

Carrageenan-induced inflammation in the murine air pouch. Mice (BALB/c; Taconic Farms Inc., Germantown, NY) were treated weekly with methotrexate $(0.5 \mathrm{mg} / \mathrm{kg})$ or a similar volume of saline intraperitoneally, followed by induction of inflammation (injection of $1 \mathrm{ml}$ of a suspension of carrageenan, $2 \% \mathrm{wt} / \mathrm{vol}$ ) in an air pouch developed on the back of the mice, as we have described previously (2). The air pouch exudate was collected, and the number of leukocytes and adenosine concentration were quantitated, as we have described (2). In other experiments, the animals received sulfasalazine $(100 \mathrm{mg} /$ $\mathrm{kg}$ ) or a similar volume of saline daily by gastric gavage during the induction of the air pouch for $3 \mathrm{~d}$. On the third day, inflammation was induced by injection of carrageenan (3).

Statistical analysis. Data were analyzed using a two-tailed Student's $t$ test with the statistical package included in EXCEL (Microsoft, Inc., Redmond, WA).

\section{Results}

To determine whether adenosine is generated extracellularly from nucleotides or is released from an intracellular store, we determined the effect of the ecto-5'-nucleotidase inhibitor APCP $(50 \mu \mathrm{M})$ on adenosine release from HMEC-1 cells exposed to stimulated neutrophils. We found, as we have reported previously for fibroblasts and human umbilical vein endothelial cells, that stimulated neutrophils promote adenosine release from methotrexate-treated HMEC-1 (Fig. 1; $P<0.01$, $n=6)$. The ecto- $5^{\prime}$-nucleotidase inhibitor APCP did not decrease significantly adenosine concentrations in supernates of control monolayers but abrogated completely the methotrexate-induced adenosine increase in supernates of methotrexatetreated HMEC-1 cells $(P<0.01, n=6)$. These findings are consistent with the hypothesis that the increased extracellular adenosine concentration present in supernatants of methotrexate-treated HMEC-1 cells is derived from nucleotides released into the extracellular space. 


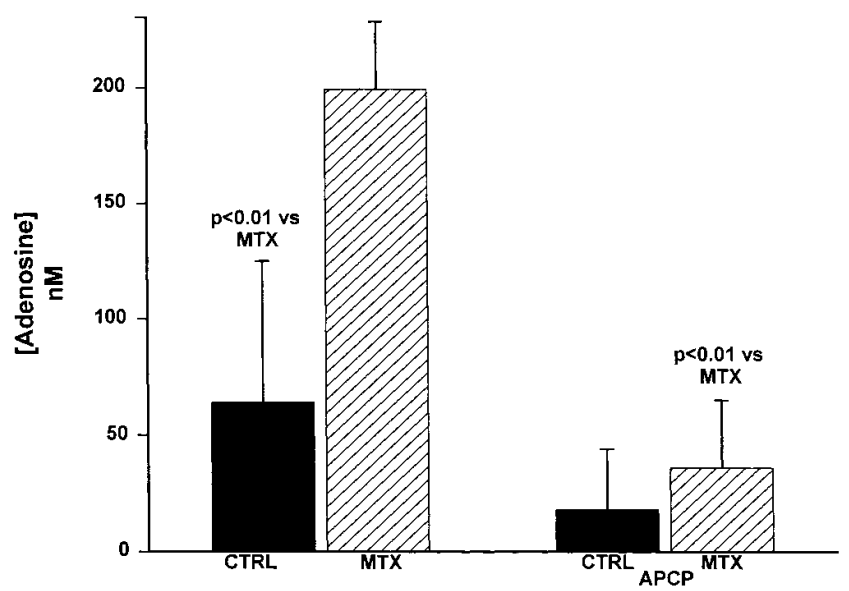

Figure 1. The effect of the ecto-5'-nucleotidase inhibitor APCP $(50 \mu \mathrm{M})$ on methotrexate $(M T X)$-mediated increases in extracellular adenosine. Subconfluent monolayers of the HMEC-1 were incubated with medium alone $(C T R L)$ or medium plus methotrexate $(0.1 \mu \mathrm{M})$ for $48 \mathrm{~h}\left(37^{\circ} \mathrm{C}, 5 \% \mathrm{CO}_{2}\right)$ until confluent. After washing, the monolayers were then exposed to stimulated (FMLP, $100 \mathrm{nM})$ neutrophils $(1.5 \times$ $10^{5}$ per well) for $2 \mathrm{~h}\left(37^{\circ} \mathrm{C}, 5 \% \mathrm{CO}_{2}\right)$ before the supernates were collected and adenosine was quantitated, as described. Shown are the results $( \pm$ SEM $)$ of six experiments.

To confirm the hypothesis that adenosine is formed extracellularly by the ecto-5' -nucleotidase-mediated dephosphorylation of AMP, we determined the effect of methotrexate on adenosine release from cells deficient in ecto-5'-nucleotidase (293T cells [12]). Because these cells adhere poorly to their substrate after exposure to stimulated neutrophils, a phenomenon which might reflect significant cellular injury, we studied the effect of oxidant stress $\left(\mathrm{H}_{2} \mathrm{O}_{2}, 100 \mu \mathrm{M}\right)$, a stress that leaves the monolayers intact, on adenosine release by $293 \mathrm{~T}$ cells. Supernatants of $293 \mathrm{~T}$ cells did not contain detectable adenosine,

Table I. Adenosine Release by 293 T Cells: The Effect of Methotrexate, $\mathrm{H}_{2} \mathrm{O}_{2}$, and Expression of Ecto-5'-nucleotidase

\begin{tabular}{|c|c|c|c|c|}
\hline & Medium & $\underset{\mathrm{H}_{2} \mathrm{O}_{2}}{\text { Medium }}+$ & $\begin{array}{l}\text { Metho- } \\
\text { trexate }\end{array}$ & $\begin{array}{c}\text { Metho- } \\
\text { trexate }+\mathrm{H}_{2} \mathrm{O}_{2}\end{array}$ \\
\hline No transfection & ND & ND & ND & ND \\
\hline Sham transfection & ND & ND & ND & ND \\
\hline $\begin{array}{l}\text { Transfection and } \\
\text { expression of } \\
\text { ecto-5'-nucleotidase }\end{array}$ & ND & ND & ND & $65 \pm 6 \mathrm{nM}^{*}$ \\
\hline
\end{tabular}

293T cells were grown to $60-70 \%$ confluence before transfection with $\mathrm{p} \beta^{\text {neo }}$ expression vector alone (sham transfection) or the $\mathrm{p} \beta^{\mathrm{NT}}$ vector containing cDNA for CD73, as described. Cells were then cultured for a further $44-48 \mathrm{~h}$ in the presence or absence of methotrexate $(1 \mu \mathrm{M})$ before washing and exposure of the cells to $\mathrm{H}_{2} \mathrm{O}_{2}(100 \mu \mathrm{M})$. After $3 \mathrm{~h}$ of incubation with medium or $\mathrm{H}_{2} \mathrm{O}_{2}$, the medium was collected, and the adenosine content was quantitated by HPLC. In these experiments, all conditions were performed in triplicate, and the results shown represent the mean $( \pm$ SEM $)$ of three different experiments. In parallel experiments, neither methotrexate nor $\mathrm{H}_{2} \mathrm{O}_{2}$ treatment increased $\mathrm{LDH}$ release $(<2 \%$ release under all conditions) from control, sham-transfected, or transfected cells. $N D$, None detected. The lower limit of detection is $5 \mathrm{nM}$. * $P<0.001$ vs. control, Student's $t$ test.

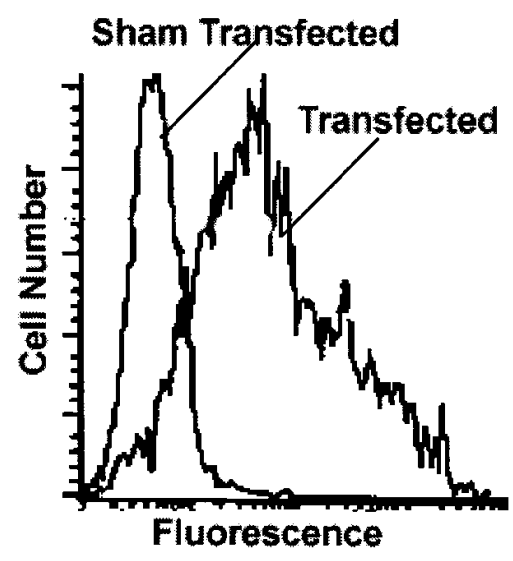

Figure 2. 293T cells transfected with ecto- $5^{\prime}-$ nucleotidase express the enzyme on their surface. 293 T cells transfected with $\mathrm{p} \beta^{\mathrm{NT}}$ expression vector containing the coding sequence of ecto-5'-nucleotidase (Transfected) or with the expression vector alone (Sham Transfected) were harvested and labeled for the expression of ecto-5'nucleotidase by immunofluorescence, as

described. Shown is a single representative cytofluorogram (of six) demonstrating surface expression of ecto-5'-nucleotidase on 293T cells. Labeling of sham-transfected cells did not differ from background or isotype control-labeled cells.

whether the cells were resting, treated with methotrexate, exposed to $\mathrm{H}_{2} \mathrm{O}_{2}$, or treated with methotrexate followed by $\mathrm{H}_{2} \mathrm{O}_{2}$ (Table I). After transfection and expression of ecto-5'-nucleotidase (optimal expression at $24 \mathrm{~h}$; Fig. 2), adenosine could be detected only in the supernate of cells pretreated with methotrexate followed by $\mathrm{H}_{2} \mathrm{O}_{2}$ treatment, but not in sham-transfected cells (Table I). These studies provide more rigorous proof of the hypothesis that adenine nucleotide, released in excess from methotrexate-treated cells, is converted extracellularly to adenosine by the action of ecto-5'-nucleotidase. Moreover, the strong correlation of the results obtained with ecto-5'-nucleotidase-deficient cells with the results obtained using the ecto-5'-nucleotidase inhibitor APCP confirms the selectivity of the inhibitor as far as adenosine production is concerned.

In previous studies, we have demonstrated that methotrexate promotes a marked increase in adenosine release into an inflammatory exudate, and that the increase of adenosine diminishes inflammation (2). To test further the hypothesis that adenosine is derived from the extracellular dephosphorylation of AMP by ecto-5'-nucleotidase, we determined the effect of the ecto-5'-nucleotidase inhibitor APCP on inflammation in the murine air pouch. As we have reported previously, methotrexate treatment promoted adenosine release $(P<0.01, n=$ $6)$ and diminished leukocyte accumulation in the murine air pouch (Fig. 3; $P<0.05, n=6$ ). Injection of APCP into the inflamed air pouch did not affect either adenosine release or leukocyte accumulation in control animals or animals treated with dexamethasone (data not shown), but abrogated completely the methotrexate-mediated increase in exudate adenosine concentration $(P<0.01, n=6)$ and decrease in leukocyte accumulation $(P<0.05, n=6)$. These results parallel the results of the in vitro experiments and confirm the hypothesis that methotrexate treatment leads to increased extracellular adenosine concentrations by a mechanism which is dependent upon the extracellular dephosphorylation of adenine nucleotides.

We have demonstrated previously that adenosine also mediates the antiinflammatory effects of sulfasalazine in the murine air pouch model of inflammation (3). Therefore, we deter- 

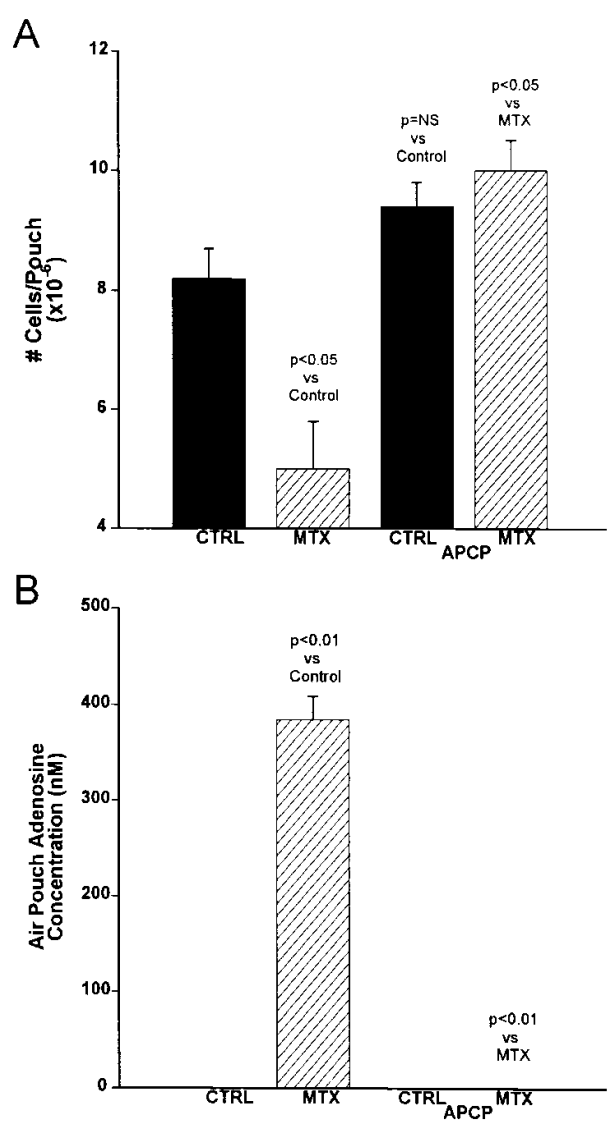

Figure 3. Injection of the ecto-5'-nucleotidase inhibitor APCP $(100 \mu \mathrm{M})$ into an inflamed air pouch reverses the antiinflammatory effects of methotrexate and diminishes methotrexate-mediated increase in exudate adenosine concentrations. BALB/c mice were treated with four weekly intraperitoneal injections of saline $(C T R L)$ or methotrexate $(M T X, 0.5 \mathrm{mg} / \mathrm{kg})$ before development of the air pouch and induction of inflammation by injection of carrageenan. In some animals, the carrageenan was suspended in saline containing the ecto-5' -nucleotidase inhibitor APCP $(100 \mu \mathrm{M})$. Shown are the mean ( \pm SEM) number of leukocytes accumulating in the inflamed air pouches $(A)$ of six animals treated as described. Also shown is the mean ( \pm SEM) exudate adenosine concentration of the exudates $(B)$ from three of these six animals.

mined whether sulfasalazine, which appears to act by the same mechanism as methotrexate, also increases extracellular adenosine by a mechanism dependent on ecto-5' -nucleotidase-mediated dephosphorylation of AMP in the murine air pouch model. As with methotrexate, inhibition of ecto-5'-nucleotidase by APCP reversed completely the sulfasalazine-mediated increase in exudate adenosine $(P<0.0007, n=6)$ and decrease in exudate leukocyte count ( $P<0.05, n=6$; Fig. 4). These results are consistent with the previous demonstration that adenosine mediates the antiinflammatory effects of sulfasalazine in the murine air pouch model of inflammation, and confirm our previous demonstration that sulfasalazine and methotrexate share an antiinflammatory mechanism.

\section{Discussion}

We report here three lines of evidence supporting the hypothesis that ecto-5'-nucleotidase activity is required for metho- trexate-mediated increases in extracellular adenosine. First, the relatively specific inhibitor of ecto-5'-nucleotidase, APCP, blocks completely the methotrexate-mediated increase in extracellular adenosine in supernates from HMEC-1 exposed to stimulated neutrophils. Second, cells that do not express ecto-5'-nucleotidase activity do not release adenosine under any conditions, but after transfection and expression of ecto-5'-nucleotidase on their surface, incubation with $\mathrm{H}_{2} \mathrm{O}_{2}$ after treatment with methotrexate does lead to adenosine release. Finally, injection of the ecto-5'-nucleotidase inhibitor APCP into the air pouch with the inflammatory stimulus prevents completely the methotrexate- and sulfasalazine-mediated release of adenosine into the inflammatory exudate, and reverses the antiinflammatory effects of methotrexate and sulfasalazine. These findings also exclude intracellular adenosine
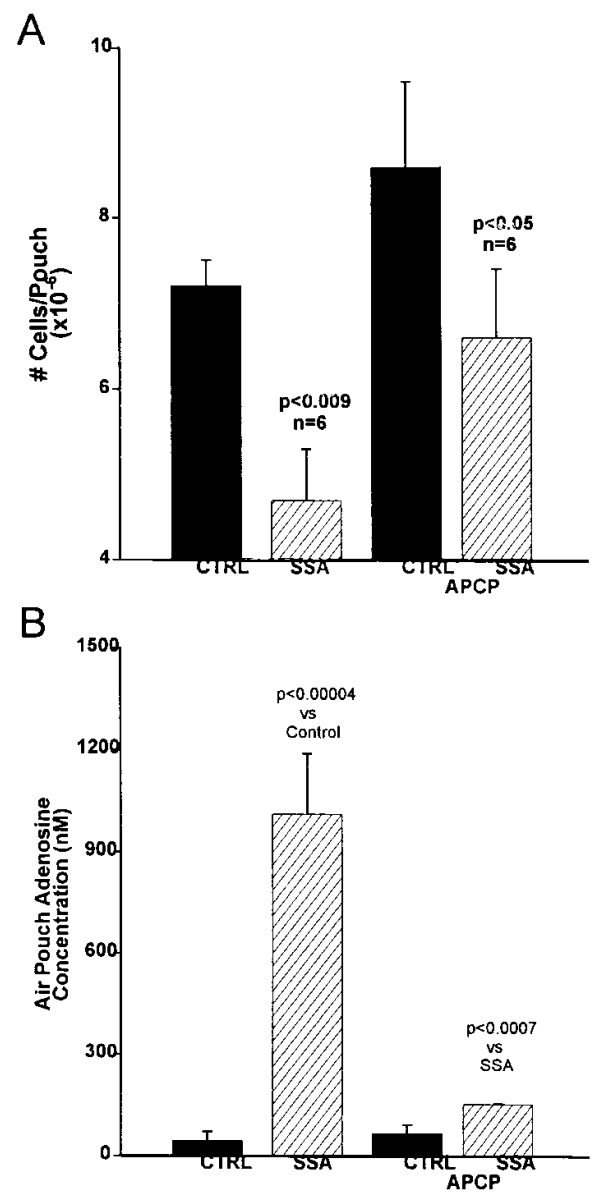

Figure 4. Injection of the ecto-5'-nucleotidase inhibitor APCP $(100 \mu \mathrm{M})$ into an inflamed air pouch reverses the antiinflammatory effects of sulfasalazine and diminishes sulfasalazine-mediated increases in exudate adenosine concentrations. BALB/c mice were treated with saline $(C T R L)$ or sulfasalazine $(S S A, 100 \mathrm{mg} / \mathrm{kg}$ ) by oral gavage for $3 \mathrm{~d}$ during the development of the air pouch. Inflammation was induced on the third day by injection of carrageenan, as described. In some animals, the carrageenan was suspended in saline containing the ecto-5'-nucleotidase inhibitor APCP $(100 \mu \mathrm{M})$. Shown are the mean $( \pm$ SEM) number of leukocytes accumulating in the inflamed air pouches $(A)$ of six animals treated as described. Also shown is the mean $( \pm$ SEM) exudate adenosine concentration of the exudates from these six animals $(B)$. 
generated as a consequence of adenosine deaminase inhibition as a source for the enhanced adenosine concentrations in the supernates of methotrexate-treated cells and the inflammatory exudates of methotrexate-treated animals.

Previous studies have demonstrated that the low levels of adenosine found in the supernatant of a variety of different cell types are derived from adenine nucleotides, but no previous studies have demonstrated that pharmacologic agents may enhance this mechanism for increasing extracellular adenosine concentrations. Thus, Kitakaze and co-workers have reported that neutrophils release adenosine which is derived primarily from the ecto-5'-nucleotidase-mediated dephosphorylation of adenine nucleotides (19). Similarly, endothelial cells release adenine nucleotides, and inhibition of ecto-5' ${ }^{\prime}$-nucleotidase increases adenine nucleotide and decreases adenosine concentrations in the supernatant of these cells (20). Both of these observations depend on the use of an enzyme inhibitor which may have other effects on adenosine and adenine nucleotide metabolism as well. Although our studies do not address the precise metabolic source of extracellular adenine nucleotide released from normal or stressed cells, our results clearly demonstrate that ecto-5'-nucleotidase-mediated conversion of adenine nucleotides is required for methotrexate and sulfasalazine to increase adenosine release.

One mechanism by which methotrexate and sulfasalazine could increase extracellular adenosine is by directly increasing adenine nucleotide release as a result of cellular injury or necrosis. We found no evidence to indicate that methotrexate, $\mathrm{H}_{2} \mathrm{O}_{2}$, or their combination were toxic to $293 \mathrm{~T}$ cells (resting or transfected) or HMEC-1 cells at the concentrations studied (LDH release). It is more likely that cell necrosis could increase adenine nucleotide release in the inflamed murine air pouches, although greater necrosis and destruction of pouch structures are, in fact, observed in the pouches of control than methotrexate- or sulfasalazine-treated animals. Moreover, there is a clear inverse correlation between exudate adenosine concentration and the number of leukocytes present in the tissue or the exudate of the air pouches $(2,3)$.

A second mechanism by which methotrexate and sulfasalazine could promote adenine nucleotide release is by promoting exocytosis of adenine nucleotide-containing intracellular vesicles. Both platelets and neurons release adenine nucleotides as a result of stimulated exocytosis, although none of the cells studied here have been shown previously to contain or release intracellular granules containing adenine nucleotides.

Another more likely explanation for the methotrexate- and sulfasalazine-mediated increase in extracellular adenosine is that methotrexate and sulfasalazine modulate purine nucleotide metabolism, and thereby promote the release of adenine nucleotides from the cells studied. Prior studies have demonstrated that human neutrophils release adenosine without exocytosis $(13,19,21)$, and the apparent quantity of adenosine released is proportional to the "energy charge" of the cells (21). Madara and colleagues have demonstrated subsequently that neutrophils directly release AMP (22), which may be converted to adenosine by the action of ecto-5' ${ }^{\prime}$-nucleotidase expressed on gut epithelial cells (19). Since methotrexate and sulfasalazine increase adenosine release from HMEC-1 and $293 \mathrm{~T}$ cells only in the presence of a noxious stimulus (stimulated neutrophils or $\mathrm{H}_{2} \mathrm{O}_{2}$ ), it is most likely that methotrexateinduced adenine nucleotide (and thus, adenosine) release is enhanced only under conditions in which the energy charge of the treated cells or tissues is lowered, as occurs in inflammation. Whatever the metabolic steps involved, the mechanism by which adenine nucleotide accumulates extracellularly is not known. Although it is most likely that adenine nucleotide diffuses or is transported across the plasma membrane, the process by which this occurs remains a matter of speculation.

It is also possible that the methotrexate- and sulfasalazinemediated increase in extracellular adenosine is due to blockade of adenosine uptake or use in the presence of a constant rate of release of adenine nucleotides. Thus, Deussen et al. (20) have reported that macrovascular endothelial cells release adenine nucleotide at a constant rate, and that the adenine nucleotide is converted extracellularly to adenosine under resting conditions. Moreover, maneuvers which enhanced adenine nucleotide release from endothelial cells or diminished adenosine use by these cells increased extracellular adenosine concentrations; a greater effect on extracellular adenosine production was observed when adenosine use was inhibited (20). However, it is unlikely that methotrexate is acting as a direct inhibitor of adenosine uptake, since methotrexate treatment increases extracellular adenosine but decreases extracellular hypoxanthine and inosine (1), a finding inconsistent with the hypothesis that purine uptake is diminished, since inosine and adenosine share a transporter. In contrast, diminished adenosine use in the presence of constant adenine nucleotide release may lead to increased extracellular adenosine concentrations, an increase which is marked at inflamed sites (23). As we have demonstrated previously, AICAR accumulates intracellularly after both methotrexate and sulfasalazine treatment $(2,3)$, and AICAR undergoes a cycle of dephosphorylation and adenosine kinase-dependent rephosphorylation similar to AMP (24). Although increased AICAR dephosphorylation-rephosphorylation has not previously been associated with cellular stress, the increased intracellular AICAR concentration present after methotrexate or sulfasalazine treatment may lead to competition with adenosine for adenosine kinase-dependent phosphorylation, and thereby diminish adenosine use. Others have reported that adenosine kinase activity is diminished under conditions of hypoxia, a phenomenon which contributes to increased adenosine release (25). Thus, although the effect of inflammation (or $\mathrm{H}_{2} \mathrm{O}_{2}$ ) on adenosine kinase activity has not been tested, it is possible that diminished adenosine use by adenosine kinase in the presence of modest increases in cellular AICAR concentration leads to a marked increase in extracellular adenosine concentration.

Both methotrexate and sulfasalazine, commonly used and effective antiinflammatory agents, diminish inflammation by promoting an increase in extracellular adenosine concentration. Our results indicate that the capacity of these antiinflammatory agents to promote an increase in extracellular adenosine concentration is completely dependent on the extracellular conversion of adenine nucleotides to adenosine. These observations suggest further that other agents that promote release of adenine nucleotides may prove to be effective for the treatment of such inflammatory diseases as rheumatoid arthritis.

\section{Acknowledgments}

The authors wish to thank Dr. Rochelle Hirschhorn for her many enlightening discussions and for reviewing this manuscript.

Dr. Montesinos is the recipient of a fellowship from the S.L.E. Foundation, Inc. This work was supported by grants from the Na- 
tional Institutes of Health (AR/AI-41911 and HL-1972 to B.N. Cronstein, AI-1220 to L.F. Thompson, and CA-6102 to R. Resta) and Pharmacia-Upjohn, Inc. This work was also supported by the General Clinical Research Center (MO1RR00096) and the Kaplan Cancer Center (CA160877).

\section{References}

1. Cronstein, B.N., M.A. Eberle, H.E. Gruber, and R.I. Levin. 1991. Methotrexate inhibits neutrophil function by stimulating adenosine release from connective tissue cells. Proc. Natl. Acad. Sci. USA. 88:2441-2445.

2. Cronstein, B.N., D. Naime, and E. Ostad. 1993. The antiinflammatory mechanism of methotrexate: increased adenosine release at inflamed sites diminishes leukocyte accumulation in an in vivo model of inflammation. J. Clin. Invest. 92:2675-2682.

3. Gadangi, P., M. Longaker, D. Naime, R.I. Levin, P.A. Recht, M.C. Montesinos, M.T. Buckley, G. Carlin, and B.N. Cronstein. 1996. The antiinflammatory mechanism of sulfasalazine is related to adenosine release at inflamed sites. J. Immunol. 156:1937-1941.

4. Cronstein, B.N. 1996. Molecular therapeutics: methotrexate and its mechanism of action. Arthritis Rheum. 39:1951-1960.

5. Chabner, B.A., C.J. Allegra, G.A. Curt, N.J. Clendeninn, J. Baram, S. Koizumi, J.C. Drake, and J. Jolivet. 1985. Polyglutamation of methotrexate. Is methotrexate a prodrug? J. Clin. Invest. 76:907-912.

6. Baggott, J.E., W.H. Vaughn, and B.B. Hudson. 1986. Inhibition of 5-aminoimidazole-4-carboxamide ribotide transformylase, adenosine deaminase and 5 -adenylate deaminase by polyglutamates of methotrexate and oxidized folates and by 5-aminoimidazole-4-carboxamide riboside and ribotide. Biochem. J. 236:193-200.

7. Baggott, J.E., S.L. Morgan, T. Ha, G.S. Alarcon, W.J. Koopman, and C.L. Krumdieck. 1993. Antifolates in rheumatoid arthritis: a hypothetical mechanism of action. Clin. Exp. Rheumatol. 11(Suppl. 8):S101-S105.

8. Barankiewicz, J., R. Jimenez, G. Ronlov, M. Magill, and H.E. Gruber. 1990. Alteration of purine metabolism by AICA-riboside in human B lymphoblasts. Arch. Biochem. Biophys. 282:377-385.

9. Gruber, H.E., M.E. Hoffer, D.R. McAllister, P.K. Laikind, T.A. Lane, G.W. Schmid-Schoenbein, and R.L. Engler. 1989. Increased adenosine concentration in blood from ischemic myocardium by AICA riboside: effects on flow, granulocytes and injury. Circulation. 80:1400-1411.

10. Barankiewicz, J., G. Ronlov, R. Jimenez, and H.E. Gruber. 1990. Selective adenosine release from human B but not $\mathrm{T}$ lymphoid cell line. J. Biol. Chem. 265:15738-15743.

11. Boyum, A. 1968. Isolation of mononuclear cells and granulocytes from human blood. Scand. J. Clin. Lab. Invest. 21(Suppl. 97):77-78.
12. Oritani, K., and P.W. Kincade. 1996. Identification of stromal cell products that interact with pre-B cells. J. Cell Biol. 134 (3):771-782.

13. Cronstein, B.N., S.B. Kramer, G. Weissmann, and R. Hirschhorn. 1983. Adenosine: a physiological modulator of superoxide anion generation by human neutrophils. J. Exp. Med. 158:1160-1177.

14. Hirschhorn, R., V. Roegner-Maniscalco, L. Kuritsky, and F.S. Rosen 1981. Bone marrow transplantation only partially restores purine metabolites to normal in adenosine deaminase-deficient patients. J. Clin. Invest. 68:13871393.

15. Resta, R., S.W. Hooker, A.B. Laurent, J.K. Shuck, Y. Misumi, Y. Ikehara, G.A. Koretzky, and L.F. Thompson. 1994. Glycosyl phosphatidylinositol membrane anchor is not required for T cell activation through CD73. J. Immunol. 153:1046-1053.

16. Gunning, P., J. Leavitt, G. Muscat, S.Y. Ng, and L. Kedes. 1987. A human beta-actin expression vector system directs high-level accumulation of antisense transcripts. Proc. Natl. Acad. Sci. USA. 84:4831-4835.

17. Sambrook, J., E.F. Fritsch, and T. Maniatis. 1989. Molecular Cloning: A Laboratory Manual. Cold Spring Harbor Laboratory, Cold Spring Harbor, NY.

18. Cronstein, B.N., S.C. Kimmel, R.I. Levin, F. Martiniuk, and G. Weissmann. 1992. A mechanism for the antiinflammatory effects of corticosteroids: the glucocorticoid receptor regulates leukocyte adhesion to endothelial cells and expression of ELAM-1 and ICAM-1. Proc. Natl. Acad. Sci. USA. 89:99919996.

19. Kitakaze, M., M. Hori, T. Morioka, S. Takashima, T. Minamino, H. Sato, M. Inoue, and T. Kamada. 1993. Attenuation of ecto-5'-nucleotidase activity and adenosine release in activated human polymorphonuclear leukocytes. Circ. Res. 73:524-533.

20. Deussen, A., B. Bading, M. Kelm, and J. Schrader. 1993. Formation and salvage of adenosine by macrovascular endothelial cells. Am. J. Physiol. 264: H692-H700.

21. Newby, A.C., C.A. Holmquist, J. Illingworth, and J.D. Pearson. 1983 The control of adenosine concentration in polymorphonuclear leucocytes, cultured heart cells and isolated perfused heart from the rat. Biochem. J. 214:317-323.

22. Madara, J.L., T.W. Patapoff, B. Gillece-Castro, S.P. Colgan, C.A. Parkos, C. Delp, and R.J. Mrsny. 1993. 5'-adenosine monophosphate is the neutrophil-derived paracrine factor that elicits chloride secretion from T84 intestinal epithelial cell monolayers. J. Clin. Invest. 91:2320-2325.

23. Cronstein, B.N., D. Naime, and G. Firestein. 1995. The antiinflammatory effects of an adenosine kinase inhibitor are mediated by adenosine. Arthritis Rheum. 38:1040-1045.

24. Vincent, M.F., F. Bontemps, and G. Van den Berghe. 1996. Substrate cycling between 5 -amino-4-imidazolecarboxamide riboside and its monophosphate in isolated rat hepatocytes. Biochem. Pharmacol. 52:999-1006.

25. Decking, U.K., G. Schlieper, K. Kroll, and J. Schrader. 1997. Hypoxiainduced inhibition of adenosine kinase potentiates cardiac adenosine release. Circ. Res. 81:154-164. 